\title{
Effects of salt ions on rheological properties of SPI-GG hybrid system
}

\author{
Bi Chonghao ${ }^{1}$, Zhang Yulai ${ }^{2}$, Wu Min ${ }^{3}$, Ni Zijian ${ }^{4}$, Li Gang ${ }^{1}$, Liu Yi ${ }^{1}$, \\ Li Dong ${ }^{3}$, Liu Yude ${ }^{1}$, Huang Zhigang ${ }^{1 *}$
}

(1. School of Materials Science and Mechanical Engineering, Beijing Technology and Business University, Beijing 100048, China;

2. School of Mechanical and Aerospace Engineering, College of Engineering, Nanyang Technological University, Nanyang 639798,

Singapore; 3. College of Engineering, National Energy $R \&$ D Center for Non-food Biomass, China Agricultural University, Beijing 100083, China; 4. Beijing University of Chemical Technology, Beijing 100029, China)

\begin{abstract}
The rheological characteristic of soy protein isolate (SPI), is one of the most important properties in its application in food industry. The effects of different concentrations of guar gum (GG, polysaccharide) and sodium chloride (salt ion) on the rheological properties of SPI were studied in this research. Steady-state shear, strain sweep and frequency sweep tests (static and dynamic rheological tests) were performed, and the following phenomenon and conclusions were drawn: (1) The viscosity of the hybrid system increases with the GG addition. This trend could also be seen with the appropriate adding of salt ion; (2) As the applied frequency increases, the storage modulus $G^{\prime}$ and the loss modulus $G^{\prime \prime}$ of the hybrid system increase at a similar rate. And the frequency sweep parameters of the hybrid system rise significantly with the increase of both GG and ionic concentration; The frequency dependence of the system varies significantly with the addition of salt ion; (3) With the concentration of the salt ion increase, the storage module $G^{\prime}$ of the hybrid system decrease indicating that salt ion destroyed the network structure of hybrid dispersion to a certain extent.
\end{abstract}

Keywords: guar gum, salt ions, soy protein isolate, rheological property, frequency dependence, food additive DOI: $10.25165 /$ j.ijabe.20171005.3245

Citation: Bi C H, Zhang Y L, Wu M, Ni Z J, Li G, Liu Y, et al. Effects of salt ions on rheological properties of SPI-GG hybrid system. Int J Agric \& Biol Eng, 2017; 10(5): 234-241.

\section{Introduction}

Soybeans are rich in protein and are always prepared into a variety of food forms, such as tofu, miso, natto, meat product and vegetarian food. Participating in essential reactions of human organism, protein is an

Received date: 2017-02-04 Accepted date: 2017-06-14

Biographies: Bi Chonghao, $\mathrm{PhD}$, research interests: material property characteristics; non-linear rheological analysis; fractal analysis, Email: bichonghao@btbu.edu.cn; Zhang Yulai, Master, research interests: non-linear rheological analysis, Email: 804968645@qq.com; Wu Min, PhD, research interests: material property characteristics, Email: wumin1283@gmail.com; Ni Zijian, Undergraduate student, research interests: dynamic rheological analysis and Fourier transform rheology, Email: 1738074793@, qq.com; Li Gang, PhD., research interests: agricultural material property characterization, Email: ligang@btbu.edu.cn; Liu Yi, PhD, important component of the human-body cells. In general, the proportion of protein in the total dry mass of the human body is approximately $18 \%^{[1]}$. Soybean protein isolate (SPI) is a type of protein food additive that can be obtained from low-temperature desolventising soybean meal as a raw material ${ }^{[2]}$. SPI contains more

research interests: characterization of agricultural material functional properties, Email: 1_y_61@hotmail.com; Li Dong, Professor, research interests: interaction between protein and polysaccharide, Email: dongli@cau.edu.cn; Liu Yude, Professor, research interests: Functional properties of food ingredients and food manufacturing machinery, Email: liuyd@th.btbu.edu.cn.

* Corresponding author: Huang Zhigang, Professor, research interests: Functional properties of food ingredients and food manufacturing machinery, Gengyun Building No.11 Fu Cheng Road Haidian District, Beijing 100048, China. Tel/Fax: +86 10 68985249, Email: huangzg@btbu.edu.cn. 
than $90 \%$ of glycinin (11S) and $\beta$-conglycinin (7S), which are the main elements containing soy protein ${ }^{[3]}$. In addition, it is a type of natural plant protein, which is rich in a variety of nutrients and has features of reducing serum cholesterol and preventing cardiopathy and cerebrovascular disease, and it may be substituted for animal protein. Therefore, SPI has become a good additive for the human body and a good nutritional supplement in food industry

SPI is a mixture of four different types of proteins. In addition, it has the following properties:

(1) Gelation

SPI can be used as a type of carrier of both water and other complexes because it has good viscosity, plasticity and elasticity, which play important roles in food processing.

(2) Emulsification

A stable emulsion can be formed when SPI meets water, and it can not only reduce the surface tension between water and air but also lower the surface tension between water and oil, which makes it a good surface-active agent.

(3)Foaming

Using the excellent foaming property in the food production process to make food softer and make it have an incredible taste, SPI is one of the best foaming agents.

During years of discovery and practice, researchers increasingly focused on the functional properties of SPI owing to its nutritional values and unique functional properties, such as gelling and emulsification ${ }^{[4,5]}$.

Due to its special characteristics, SPI has been used not only in fermented food but also in nutritious food and in other food products. Additionally, SPI is also a common material that can be used in meat, dairy and flour products. SPI can improve the taste of food, increase its nutritive value, lower cholesterol and prevent heart and artery diseases. That is why the application of SPI in the food industry is gradually increasing. There are multiple factors affecting the rheological properties of SPI, e.g. temperature, $\mathrm{pH}$, ionic strength and the polysaccharide. In addition, some features of SPI can be improved by changing the process parameters and adding some other materials, such as ionic compounds and polysaccharides $^{[4,6]}$. Due to the unique structural and functional properties of proteins that ubiquitously exist in the modern food industry and the interaction between proteins and polysaccharides, SPI and polysaccharides are important for developing the desired texture ${ }^{[7,8]}$. Protein-polysaccharide interactions can be used to design and develop new food products ${ }^{[8,9]}$.

Polysaccharides comprise multiple monosaccharide molecules, which are a kind of carbohydrate. They are widely distributed in nature and play an important role in many basic life activities, such as constituting the composition of animals and plants, functioning as anticoagulant material in the human body and acting as the nutrients stored in animals and plants. The structural unit of polysaccharides is the monosaccharide, which generally does not dissolve in water, has no sweet taste and cannot form crystals but can be hydrolysed. Guar gum (GG, polysaccharide) is a branched structure comprising 1,4-linked $\beta$-d-mannopyranosyl units (Man) and 1,6-linked $\alpha$-d-galactopyranosyl units (Gal) of polysaccharides $^{[10-14]}$. The molecular weight of GG has been reported to be $\sim 2.8 \times 10^{7} \mathrm{~g} / \mathrm{mol}^{[15,16]}$. Owing to its good characteristics, including low cost, non-toxic, high viscosity and high water solubility, GG is now used as a thickening, emulsifying and stabilising additive in many industries ${ }^{[17,18]}$. Moreover, GG in the food industry plays an excellent role as a stabiliser due to its thickening and emulsifying effects. In particular, in flour products, GG also exhibits good properties, such as preventing sticking, staying hydrated, increasing flour strength, extending the shelf life and maintaining excellent food quality. Furthermore, GG can be used as a condiment, optimising the sensory quality of food, including the texture and structure of the product and its rheological properties. In general, GG is a versatile additive that is widely used in the food industry.

Because of the irreplaceable functional properties of proteins and polysaccharides, they are playing more and more important roles in modern food manufacturing. Interactions between proteins and polysaccharides in food system have also been research hotspots in recent years ${ }^{[7,8]}$. With increasing demand for food products with this desired texture, proteins and polysaccharides 
play vital roles owing to their functional properties and interaction capability ${ }^{[19]}$. Therefore, it is necessary to understand the interaction between proteins and polysaccharides systematically to meet the market demand. Specific functional properties of SPI can be improved by engineering technology or using additives, such as ions and polysaccharides ${ }^{[6,20]}$. Therefore, in this investigation, GG, salt ions and SPI hybrid system will be used as a main research object to investigate the effects of salt ions on the linear rheological properties of SPI.

\section{Materials and methods}

\subsection{Materials and equipment}

Commercial soy protein isolate (protein content $>90 \%$ ) was obtained from Messenger Biotechnology Co., Ltd. as a gift. Guar gum (AR grade) was bought from Source Leaf Biotechnology Co., Ltd. Sodium chloride (AR grade) was purchased from Beijing Yinghai Fine Chemical Co., Ltd.

Rheometer (AR2000 ex); TA Instruments Company (USA); Magnetic stirrer (85-2); Jiangsu Jintan Jingda Instrument Factory (China); Analytical Balance (AL 204); Mettler-Toledo (Switzerland); Precision power by electric mixer (JJ-1); Changzhou Guohua Electric Equipment Co. Ltd (Jiangsu province, China).

\subsection{Sample preparation}

Firstly, $9 \mathrm{~g}$ of SPI powder was added into $100 \mathrm{~mL}$ of deionised water by stirring the solution for $20 \mathrm{~min}$ to completely dissolve the powder. Three more beakers were used to formulate three solutions with different concentrations by pouring $50 \mathrm{~g}$ of deionised water and dissolving $0.1875 \mathrm{~g}, 0.15 \mathrm{~g}$ and $0.1125 \mathrm{~g}$ of GG powder, respectively. Next, the solutions were stirred for $20 \mathrm{~min}$. Then, the SPI solution was mixed with the three GG solutions by stirring for $20 \mathrm{~min}$ to formulate three solutions with different concentrations $(0.125 \%, 0.1 \%$ and $0.075 \%$, respectively). Then, another beaker was used in which $100 \mathrm{~g}$ of deionised water was mixed with $6 \mathrm{~g}$ of SPI powder and stirred for $20 \mathrm{~min}$. Finally, these four samples were maintained at room temperature for $12 \mathrm{~h}$.

Identical to the abovementioned procedure, another three beakers were used in which $100 \mathrm{~g}$ of deionised water was mixed with $9 \mathrm{~g}$ of SPI powder and stirred for 20 min to fully dissolve the SPI powder. Then, using another three beakers, were formulated three cups of the same solution were formulated by adding $50 \mathrm{~g}$ of deionised water and dissolving $0.1875 \mathrm{~g}$ of GG powder, and stirring the solutions for $20 \mathrm{~min}$. Next, the three same solutions of GG were mixed with the three readied SPI solutions. Stirring each for $20 \mathrm{~min}$ and then adding $0.5 \mathrm{~g}, 0.25 \mathrm{~g}$ and $0.125 \mathrm{~g}$, respectively, of $\mathrm{NaCl}$ into the SPI-GG hybrid system, they were each stirred for $20 \mathrm{~min}$.

Before the rheological test, all the overnight samples were stirred for $1 \mathrm{~min}$ using a magnetic stirrer before sampling.

\subsection{Rheological test}

The tests of rheological properties were all conducted on an AR2000ex rheometer and the temperature was kept at $25^{\circ} \mathrm{C}$, controlled by a Peltier System. Aluminium parallel plate geometry (40 $\mathrm{mm}$ diameter) was chosen for the dynamic rheological test. The gap between the geometry and the Peltier was $1000 \mu \mathrm{m}$.

The characterisation of the rheological properties consisted of these three tests: steady shear test, strain sweep test and frequency sweep test. Each test was performed in consist temperature and in temperature-changing process.

\subsection{Strain sweep test}

The temperature of the strain sweep test was controlled at $25^{\circ} \mathrm{C}$ and the frequency was set to $1 \mathrm{~Hz}$. Testing the storage modulus $G^{\prime}$ and the loss modulus $G^{\prime \prime}$ of the samples, the strain increased to determine the Linear Viscoelastic Region (LVR) of each sample.

The oscillation stain in the dynamic rheological test was controlled in the LVR, which is the area where the stain imposed on the samples was relatively small, so that the internal structure of the sample was not be affected by oscillation.

\subsection{Frequency sweep test}

The frequency sweep test was performed at the end of the strain sweep test, since the oscillatory strain amplitude of this test must be within the LVR of each sample. The temperature of the experiment was controlled at $25^{\circ} \mathrm{C}$, and the frequency was set at $1 \mathrm{~Hz}$. The setting strain was controlled at $0.2 \%$, and both the 
storage modulus $G^{\prime}$ and the loss modulus $G^{\prime \prime}$ of the SPI-GG hybrid system were detected through observing the corresponding frequency scanning curve. The frequency dependence of both storage modulus $\left(G^{\prime}\right)$ and loss modulus $\left(G^{\prime \prime}\right)$ of the SPI-GG hybrid system could be fitted by the power law model:

$$
\begin{aligned}
& G^{\prime}=K^{\prime} \cdot f^{n^{\prime}} \\
& G^{\prime \prime}=K^{\prime \prime} \cdot f^{n^{\prime \prime}}
\end{aligned}
$$

The parameters $K^{\prime}$ and $K^{\prime \prime}$ in the above equations are the power law constants whose dimensions are $\mathrm{Pa} \cdot \mathrm{s}^{\mathrm{n}} / \mathrm{rad}^{\mathrm{n}}$. The letters $n^{\prime}$ and $n^{\prime \prime}$ in the equations are the frequency index, which reflects the viscoelasticity frequency dependence of the samples ${ }^{[21]}$.

\subsection{Statistical analysis of the experimental data}

In this paper, the data are shown as averages. All the above experiments were repeated three times, and the average values were shown in tables and figures using Excel software to draw the corresponding curve.

\section{Results and discussion}

\subsection{Steady shear test}

The effect of shear rate on the viscosity $(\eta)$ of the SPI-GG hybrid system with different concentrations of GG at $25^{\circ} \mathrm{C}$ is shown in Figure 1. The diagram illustrates that within the shear rate range of the steady shear test (1-100 s $\mathrm{s}^{-1}$, the viscosity of all samples decrease with the increasing shear rate. The effect of the GG concentration on the viscosity of the system is not obvious but indicates the shear thinning properties of the system, which is relevant to the arrangement of the molecular structure along the shear direction. The effect of the lower shear rate on the molecule network structure is not significant, which indicates that at low shear rate, the change of molecule network structure is to a low extent. To maintain the molecular network structure, the molecules provide resistance to deformation that should have been from shear. So, the viscosity of the SPI solution is higher at lower shear rate. At higher shear rates, the molecular network structure will be damaged under the action of shear and subsequently the dispersed molecules will be arranged in the direction of the shear force, which greatly reduces the molecular anti-shear behaviour, thereby reducing the viscosity ${ }^{[22]}$.

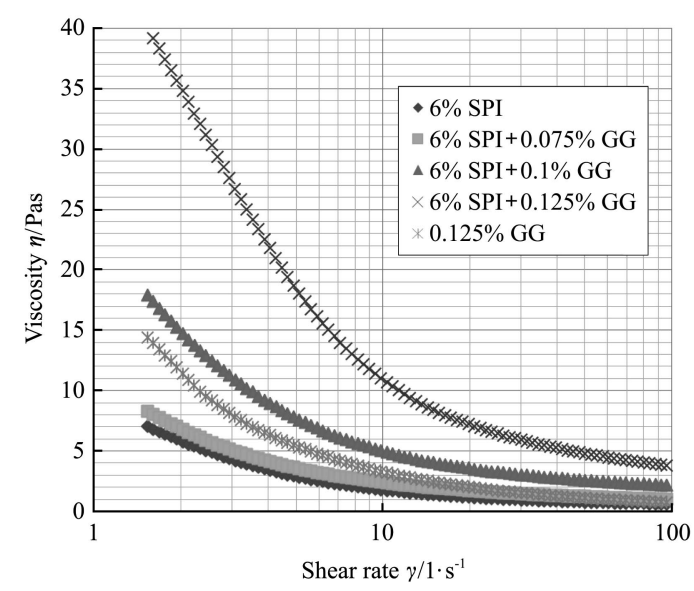

Figure 1 Effects of shear rate on the viscosity $\eta$ of the soy protein isolate (SPI)-guar gum (GG) hybrid system with different GG concentrations $\left(t=25^{\circ} \mathrm{C}\right)$

As shown in Figure 1, the viscosities of both the pure protein solution and the pure gum solution are significantly less than that of the SPI-GG hybrid system, which indicates that the protein and polysaccharide have a significant cross-linking and synergistic effect in viscosity property.

At $25^{\circ} \mathrm{C}$, the effects of the ionic concentration on the viscosity of the SPI-GG hybrid system with shear rates are shown in Figure 2. From Figure 2, it is observed that within the steady shear rate ranging from $1 \mathrm{~s}^{-1}$ to $100 \mathrm{~s}^{-1}$ the viscosity decreases with increasing shear rate, which shows obvious shear thinning properties, except for the sample that was treated with the highest ionic $(0.5 \%)$ concentration. The higher the ionic concentration is, the lower the viscosity of the SPI-GG hybrid system is. This phenomenon indicates that salt ion destroyed part of the cross-linking and synergistic effect between protein and polysaccharide.

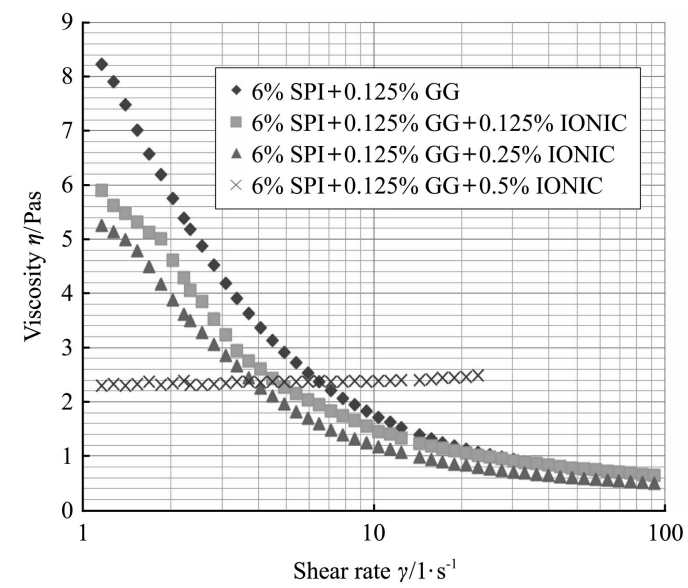

Figure 2 Effects of shear rate on the viscosity of the SPI-GG hybrid system with different ionic concentration $\left(t=25^{\circ} \mathrm{C}\right)$ 
However, the viscosity of the sample whose ionic concentration is $0.667 \%$ did not change significantly with the increase in shear rate, illustrating the characteristics of Newtonian fluid.

\subsection{Frequency sweep test}

In the dynamic rheological test, the rheological profiles of the samples are expressed as a change of dynamic modulus $G^{\prime}$ and $G^{\prime \prime}$ through frequency changes. The variable, $G^{\prime}$ is known as the elastic modulus or storage modulus, which is used to describe the character of the material similar to the solid, while $G^{\prime \prime}$ is the viscous modulus or loss modulus, which is used to describe the character of the material, similar to the liquid. Figure 3 shows the trend of $G^{\prime}$ and $G^{\prime \prime}$ of SPI-GG hybrid system with the frequency ranging from $0.3-1.0 \mathrm{~Hz}$ at $25^{\circ} \mathrm{C}$ and $0.2 \%$ strain. From Figure 3, it can be seen that the $G^{\prime}$ and $G^{\prime \prime}$ values of all the hybrid samples increase with the frequency increase, however, the trend of $G^{\prime}$ of SPI and GG alone sample shows a negative correlation with frequency. When the frequency is constant, $G^{\prime}$ of the hybrid system gradually increases with the increase of GG concentration, which means that the elasticity of the sample increases with the addition of GG. From Figure 3 , we can clearly see that $G^{\prime}$ values are higher than $G^{\prime \prime}$ values among all the samples, which indicates that these samples have the structural characteristics of a semi-solid and biopolymers ${ }^{[22]}$.

The frequency sweep curve of the hybrid system can be well fitted by the power law model. Table 1 summarises the effects of different concentrations of GG on the power law model parameters of the SPI hybrid system. It can be seen from the table that $G^{\prime}$ and $G^{\prime \prime}$, along with the frequency trends are well fitted to the power law model $\left(R^{2}>0.96\right)$. The $K^{\prime}$ and $K^{\prime \prime}$ values of hybrid system vary significantly when GG added into the system. Both of the value of $K^{\prime}$ and $K^{\prime \prime}$ increase with the concentration of GG increase which confirms the previous view that the network structure of the hybrid system increases with the addition of GG.

As the GG concentration increases from 0 to $0.125 \%$, the value of $n^{\prime}$ gradually increases, ranging from -0.033 to 0.1851 . This indicates that the elastic frequency dependence of the hybrid system gradually increases with the addition of GG and the relationship between them $\left(G^{\prime}\right.$ and $f$ ) gradually changes from one of negative correlation to one of positive correlation. At the same time, the value of $n^{\prime \prime}$ decreases, which indicates that the viscosity frequency dependence of the hybrid system decreases with the addition of GG.
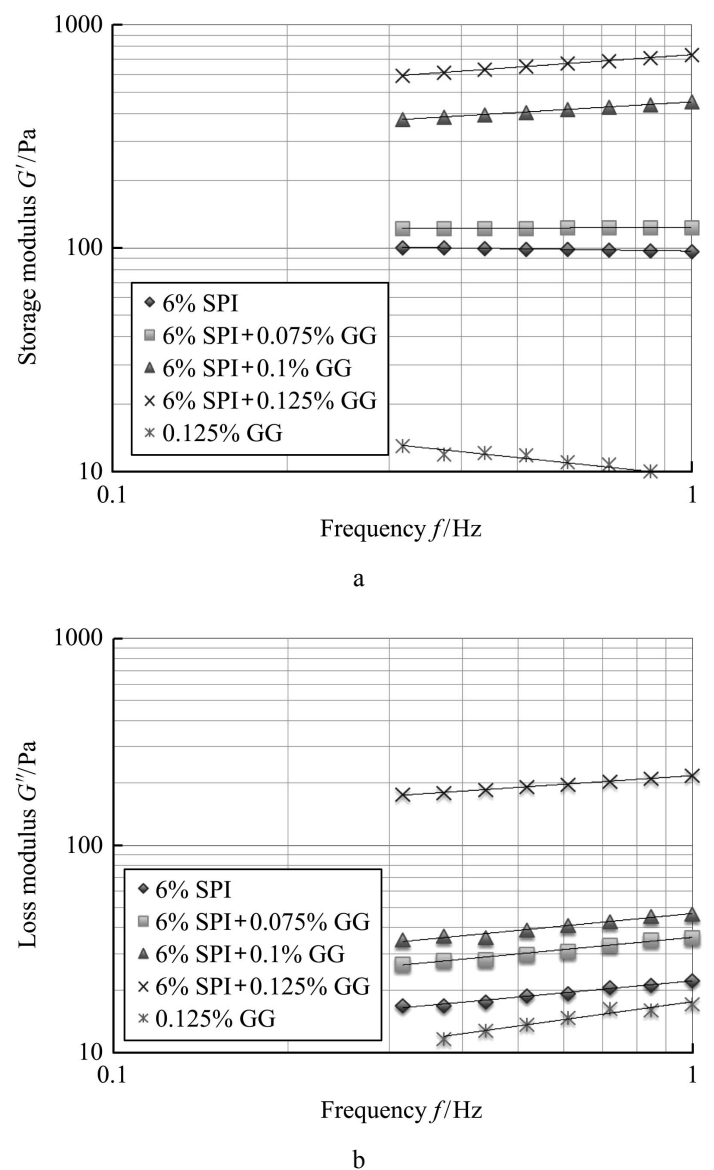

Figure 3 Effects of frequency on the frequency sweep parameters of the SPI-GG hybrid system with different GG concentrations $\left(t=25^{\circ} \mathrm{C}\right.$ and $\left.\gamma=0.2 \%\right)$

Table 1 Effects of guar gum (GG) concentration on the power law model parameters of the soy protein isolate (SPI)-GG hybrid system

\begin{tabular}{cccccccc}
\hline \multirow{2}{*}{ Samples' name } & \multicolumn{3}{c}{$G^{\prime}=K^{\prime} \cdot f^{n^{\prime}}$} & & \multicolumn{3}{c}{$G^{\prime \prime}=K^{\prime \prime} \cdot f^{n^{\prime \prime}}$} \\
\cline { 2 - 4 } \cline { 7 - 8 } & $K^{\prime}$ & $n^{\prime}$ & $R^{2}$ & & $K^{\prime \prime}$ & $n^{\prime \prime}$ & $R^{2}$ \\
\hline 6\% SPI & 96.993 & -0.0330 & 0.99 & 22.149 & 0.2603 & 0.98 \\
6\% SPI+0.075\% GG & 123.61 & 0.0069 & 0.99 & 35.958 & 0.2667 & 0.98 \\
6\% SPI+0.1\% GG & 451.54 & 0.1570 & 0.99 & 46.88 & 0.2714 & 0.97 \\
6\% SPI+0.125\% GG & 734.65 & 0.1851 & 0.99 & 217.26 & 0.1892 & 0.99 \\
0.125\% GG & 9.5351 & -0.2730 & 0.926 & 17.74 & 0.4201 & 0.96 \\
\hline
\end{tabular}

Figure 4 is frequency sweep profiles of samples at $25^{\circ} \mathrm{C}$ and $0.2 \%$ strain for $G^{\prime}$ and $G^{\prime \prime}$, respectively. The $G^{\prime}$ values of all samples increased or decreased in low amplitude with increasing frequency. When the salt ion concentration is below $0.167 \%$, the relation between $G^{\prime}$ 
and $f$ is positive correlation. However, when the salt ion concentration is over $0.330 \%$, the relationship gradually changes from positive correlation to negative correlation. When the frequency is constant, the value of $G^{\prime}$ of the hybrid system decreases gradually with the increasing ionic concentration, which means that the elasticity property of the hybrid system decreases with the addition of salt ions. This phenomenon indicates that the frequency dependence of elasticity of hybrid system is complex and significant, and depends on the concentration of salt ion heavily. The viscosity of the SPI-GG hybrid system without ionic treatment is higher than that with the ionic-treated SPI-GG hybrid system. Also, the viscosity of the samples decreases with increasing ionic concentration. The frequency sweep curve of the hybrid system can be well fitted with the power law model. Table 2 summarises the effect of different ionic concentrations on the power law model parameters of the SPI-GG hybrid system.
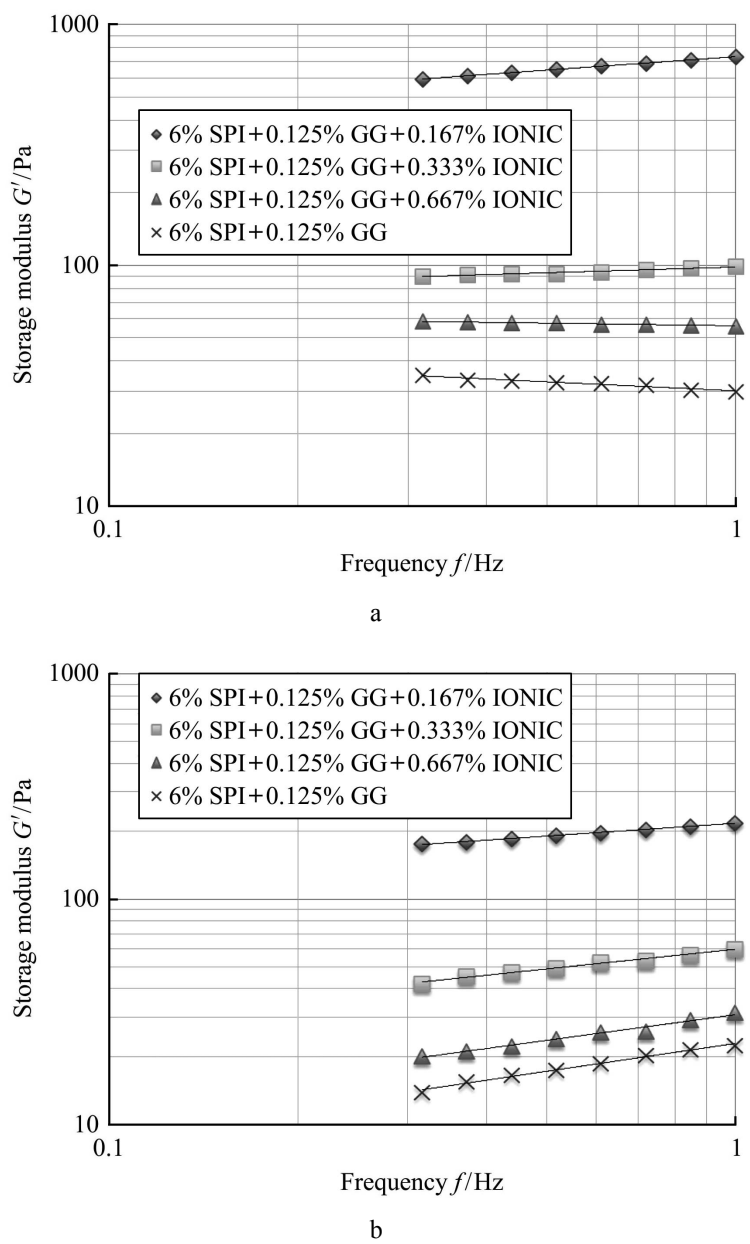

Figure 4 Effects of frequency on the frequency sweep parameters of the SPI-GG hybrid system with different ionic concentrations $\left(t=25^{\circ} \mathrm{C}\right.$ and $\left.\gamma=0.2 \%\right)$
Table 2 Effects of ionic concentration on the power law model parameters of the SPI-GG hybrid system

\begin{tabular}{cccccccc}
\hline \multirow{2}{*}{ Samples' name } & \multicolumn{3}{c}{$G^{\prime}=K^{\prime} \cdot f^{n^{\prime}}$} & & \multicolumn{3}{c}{$G^{\prime \prime}=K^{\prime \prime} \cdot f^{n^{\prime \prime}}$} \\
\cline { 2 - 5 } \cline { 7 - 8 } & $K^{\prime}$ & $n^{\prime}$ & $R^{2}$ & & $K^{\prime \prime}$ & $n^{\prime \prime}$ & $R^{2}$ \\
\hline SPI+GG & 734.65 & 0.1851 & 0.99 & 217.26 & 0.1892 & 0.99 \\
SPI+GG+0.167\% IONIC & 98.327 & 0.079 & 0.96 & 59.914 & 0.2908 & 0.99 \\
SPI+GG+0.333\% IONIC & 56.071 & -0.036 & 0.96 & 30.673 & 0.3783 & 0.98 \\
SPI+GG+0.667\% IONIC & 30.108 & -0.124 & 0.97 & 22.864 & 0.4112 & 0.99 \\
\hline
\end{tabular}

It can be seen from the table that the changing trends of $G^{\prime}$ and $G^{\prime \prime}$ along with the changing trend of angular frequency are in good agreement with the power law model $\left(R^{2}>0.96\right)$. The values of $K^{\prime}$ and $K^{\prime \prime}$ of the samples without ionic treatment are significantly higher than those of ionic-treated samples. This is because the GG treatment affects the SPI to form the good network structure. With increasing ionic concentration, $n^{\prime \prime}$ gradually increases, while $n^{\prime}$ declines even to a negative value.

\subsection{Strain sweep test}

Figure 5 shows the effect of different concentrations of GG on $G^{\prime}$ (at different strain) of the SPI-GG hybrid system. From Figure 5, the linear viscoelastic region of the system ranges approximately from 0.1 to 1 . The storage modulus $G^{\prime}$ displays a decreasing trend with increasing strain. The effect of GG on $G^{\prime}$ of the SPI solution is significant and the higher the GG concentration, the higher the value of $G^{\prime}$. It is noted that the $G^{\prime}$ value of SPI and GG are both below $100 \mathrm{~Pa}$, however, the value of $G^{\prime}$ of their hybrid system reaches over $500 \mathrm{~Pa}$. This indicates a very strong interaction and molecular entanglement between SPI and GG.

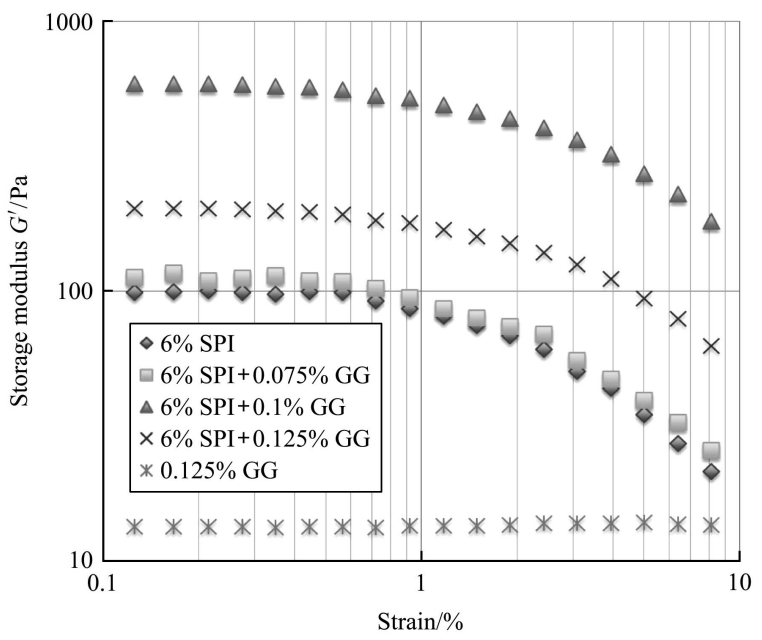

Figure 5 Effects of strain on the $G^{\prime}$ of the SPI-GG hybrid system with different $\mathrm{GG}$ concentrations $\left(t=25^{\circ} \mathrm{C}\right)$ 
Figure 6 shows the effects of different ionic concentrations on $G^{\prime}$ (at different strain) of the SPI-GG hybrid system. As shown, the additions of salt ions decrease the $G^{\prime}$ value of hybrid system significantly (from $691 \mathrm{~Pa}$ to $\sim 100 \mathrm{~Pa}$ ). However, the salt ions content does not affect the elastic property of hybrid system significantly. The results showed that salt ion affects the elasticity property of hybrid system in a significant way. This may due to the salt ion disengaged part of the molecular entanglement. Moreover, the higher content of salt ion exists, the more the molecular entanglement disaggregates.

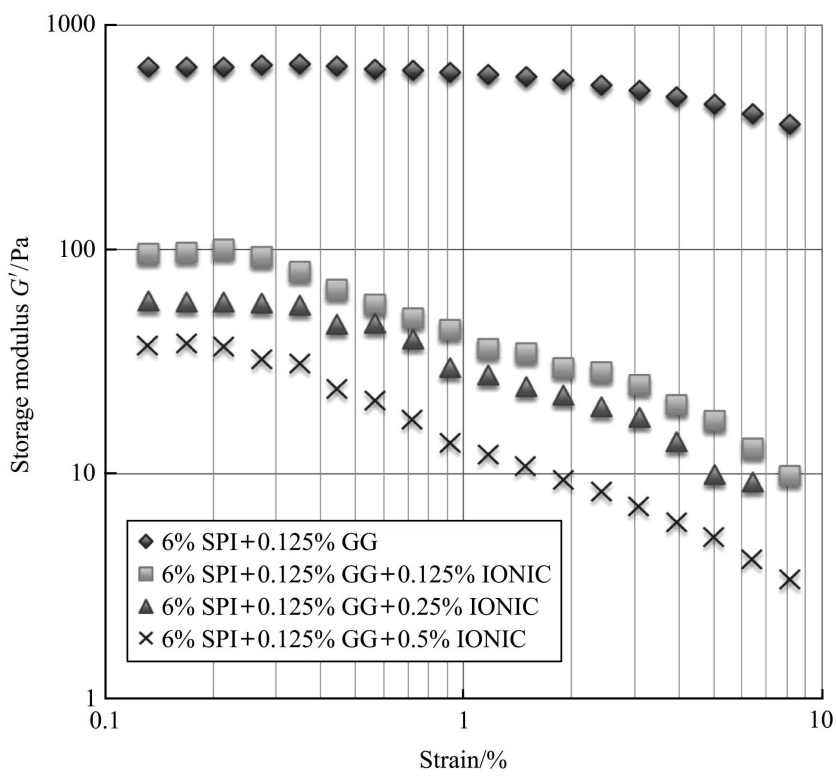

Figure 6 Effects of strain on the $G^{\prime}$ of the SPI-GG hybrid system with different ionic concentrations $\left(t=25^{\circ} \mathrm{C}\right)$

\section{Conclusions}

In this research, the effects of different concentrations of GG on the rheological properties of SPI and different ionic concentrations on the SPI-GG hybrid system were investigated.

It is found that the protein and polysaccharide have a significant cross-linking and synergistic effect in both viscosity and elastic properties. However, salt ion with a very low concentration may strongly destroyed part of the cross-linking and synergistic effect between protein and polysaccharide. The network structure of the hybrid system increases with the addition of GG. Viscosity frequency dependence of the hybrid system decreases with the addition of GG. The frequency dependence of elasticity of hybrid system is complex, and depends on the concentration of salt ion heavily. There may be a very strong interaction and molecular entanglement between SPI and GG, while low dose salt ions are able to disengaged part of the molecular entanglement effectively.

\section{Acknowledgement}

This research was supported by the Research Foundation for Youth Scholars of Beijing Technology and Business University (QNJJ2016-17). This research project was also supported by the National Natural Science Foundation of China (31301593) and Research and Development Fund for University's Doctoral Discipline of China (20130008120021).

\section{[References]}

[1] Huysveld S, de Meester S, Peiren N, Muylle H, Lauwers L, Dewulf J. Resource use assessment of an agricultural system from a life cycle perspective - a dairy farm as case study. Agricultural Systems, 2015; 135: 77-89.

[2] Aguirre F J S, Milesi V, Añón M C. Effect of extraction and precipitation conditions during soybean protein isolate production on the genistein series content. Journal of the American Oil Chemists Society, 2007; 84(3): 305-314.

[3] Petruccelli S, Anon M C. Soy protein isolate components and their interactions. Journal of Agricultural \& Food Chemistry, 1995; 43(7): 1762-1767.

[4] Bi C H, Li D, Wang L J, Adhikari B. Viscoelastic properties and fractal analysis of acid-induced SPI gels at different ionic strength. Carbohydrate Polymers, 2013; 92(1): 98-105.

[5] Maltais A, Remondetto G E, Gonzalez R, Subirade M. Formation of soy protein isolate cold-set gels: protein and salt effects. Journal of Food Science, 2010; 70(1): C67-C73.

[6] Bi C H, Li D, Wang L J, Adhikari B. Effect of LBG on the gel properties of acid-induced SPI gels. LWT - Food Science and Technology, 2017; 75: 1-8.

[7] Corredig M, Sharafbafi N, Kristo E. Polysaccharide-protein interactions in dairy matrices, control and design of structures. Food Hydrocolloids, 2011; 25(8): 1833-1841.

[8] Perez A A, Carrara C R, Sánchez C C, Patino J M R, Santiago L G. Interactions between milk whey protein and polysaccharide in solution. Food Chemistry, 2009; 116(1): 104-113.

[9] Alvarez M D, Fernández C, Olivares M D, Canet W. A rheological characterisation of mashed potatoes enriched with soy protein isolate. Food Chemistry, 2012; 133(4): $1274-1282$. 
[10] Dea I C M, Morrison A. Chemistry and interactions of seed galactomannans. Advances in Carbohydrate Chemistry \& Biochemistry, 1975; 31(8): 241-312.

[11] Dey P M. Biochemistry of $\alpha$-D-Galactosidic Linkages in the Plant Kingdom. Advances in Carbohydrate Chemistry \& Biochemistry, 1980; 37: 283-372.

[12] Dierckx I S, Dewettinck I K. Seed Gums. Biopolymers Online. Wiley-VCH Verlag $\mathrm{GmbH} \&$ Co. KGaA, 2005; 321-343.

[13] Todd P A, Benfield P, Goa K L. Guar gum. Drugs, 1990; 39(6): 917-928.

[14] Santos R, Gomes D, Macedo H, Barros D, Tibério C, Veiga, $\mathrm{A} \mathrm{S}$, et al. Guar gum as a new antimicrobial peptide delivery system against diabetic foot ulcers Staphylococcus aureus isolates. Journal of Medical Microbiology, 2016; 65(10): 1092-1099.

[15] Barth H G, Smith D A. High-performance size-exclusion chromatography of guar gum. Journal of Chromatography A, 1981; 206(2): 410-415.

[16] Vijayendran B R, Bone T. Absolute molecular weight and molecular weight distribution of guar by size exclusion chromatography and low-angle laser light scattering. Carbohydrate Polymers, 1984; 4(4): 299-313.

[17] Chudzikowski R J. Guar gum and its application. J Soc Cosmet Chem, 1971; 22(1): 43.

[18] Wang Q, Ellis P R, Ross-Murphy S B. The stability of guar gum in an aqueous system under acidic conditions. Food Hydrocolloids, 2000; 14(2): 129-134.

[19] Foegeding E A. Rheological properties of whey protein isolate gels determined by torsional fracture and stress relaxation. Journal of Texture Studies, 2010; 23(3): 337-348.

[20] Bi C H, Li D, Wang L J, Wang Y, Adhikari B. Characterization of non-linear rheological behaviour of SPI-FG dispersions using LAOS tests and FT rheology. Carbohydrate Polymers, 2013; 92(2): 1151-1158.

[21] Papagiannopoulos A, Sotiropoulos K, Stergios P. Particle tracking microrheology of the power-law viscoelasticity of xanthan solutions. Food Hydrocolloids, 2016; 61: 201-210.

[22] Oh M, So J, Yang S. Rheological evidence for the silica-mediated gelation of xanthan gum. Journal of Colloid and Interface Science, 1999; 216(2): 320-328. 